\title{
Kasuistik - wichtiges Element der Fortbildung
}

Es in guter Arzt muss beides beherrschen, die Kenntnis der wissenschaftlichen Grundlagen seines Fachgebiets ebenso wie die handwerklichen und auch kunsthandwerklichen Aspekte der Medizin. Zu Letzteren gehört neben einer hohen Fähigkeit zur Kommunikation und Empathie auch das Abrufen von Erfahrungen und Erinnerungen. Eine einmal erlebte interessante Diagnose oder eine ungewöhnliche Symptomkonstellation mit einer nicht dazu passenden Diagnose hilft beim nächsten Auftreten der gleichen Situation ungemein viel mehr als alle Kenntnisse von Leitlinien oder Statistiken. Das Wiedererkennen ist ein zentrales Element des sog. klinischen Blicks und begründet die Notwendigkeit einer ,Erfahrungshierarchie“ in der Medizin. Diese darf nicht auf andere Aspekte insbesondere der wissenschaftlichen Seite der Medizin übergreifen, sie muss aber im Interesse der Patienten bezüglich der Krankenversorgung erhalten bleiben.

Der diesjährige 116. Kongress der Deutschen Gesellschaft für Innere Medizin betont die fallorientierte Fort- und Weiterbildung genau aus diesem Grunde, und es ist daher naheliegend, auch das Kongressheft der gesellschaftsassoziierten Zeitschrift kasuistisch auszurichten. Erfreulicherweise hat die Mehrzahl der angefragten Kollegen, meist Leiter klinischer Einrichtungen an großen universitären oder Versorgungskrankenhäusern, die Einladung, eine Kasuistik für dieses Heft beizusteuern, aufgegriffen. Ich freue mich daher sehr, Ihnen dieses Heft in der Zeitschrift mit einer ausschließlichen Fülle von spannenden kasuistischen Darstellungen, die mir selbst in drei Fällen bereits jetzt im Alltag geholfen haben, präsentieren zu dürfen. Das Heft enthält zudem die Rede anlässlich des festlichen Abends des Kongresses am 11. 4. 2010, die sich u.a. ebenfalls mit dem Aspekt des Kunsthandwerks im Verhältnis zur Wissenschaft beschäftigt.

Ich wünsche Ihnen allen viel Freude beim Lesen, Gewinn im Alltag durch Kenntnis der beschriebenen, in meinen Augen spannenden Fälle und zudem eine gewinnbringende Teilnahme an unserem Kongress.

Jürgen Schölmerich 